\title{
Dor em Crianças com Deficiências Cognitivas: uma Revisão Sobre os Instrumentos de Avaliação Comportamentais
}

\author{
Pain in Children with Cognitive Disabilities: a Review of Behavioral Assessment Tools
}

\author{
Andressa Veruska Robes ${ }^{\text {a }}$ Paulo Henrique Perlatti D’Alpino*ab
}

\begin{abstract}
${ }^{a}$ Universidade Anhanguera de São Paulo, Programa de Pós-Graduação Stricto Sensu em Ensino de Ciências e Saúde. SP, Brasil. bUniversidade Anhanguera de São Paulo, Programa de Pós-Graduação Stricto Sensu em Biotecnologia e Inovação em Saúde. SP, Brasil. *E-mail: paulodalpino@gmail.com
\end{abstract}

\begin{abstract}
Resumo
Crianças com deficiências cognitivas correm maior risco de sentir dor. Além disso, demonstrou-se que essa população pediátrica, frequentemente, recebe tratamento inadequado da dor. A dor é, muitas vezes, muito difícil de avaliar, especialmente, em um subgrupo definido com deficiência intelectual, não verbal ou ainda com deficiência cognitiva grave. Embora o autorrelato seja uma alternativa para um pequeno número de crianças com menor acometimento intelectual, as medidas observacionais têm as evidências mais consistentes para apoiar seu uso de escalas comportamentais. Consequentemente, várias ferramentas observacionais de avaliação da dor foram propostas para superar esse problema. Em função da ausência de uma ferramenta de avaliação ideal, a avaliação precisa da dor requer, após uma análise caso a caso, a seleção de um instrumento mais apropriado ou uma associação de instrumentos. O objetivo deste trabalho é fornecer uma revisão dos instrumentos e escalas de avaliação da dor comumente usadas em crianças com deficiência cognitiva. Espera-se que com a discussão crítica dos recursos próprios de cada escala, bem como de sua aplicabilidade clínica esses possam, de certa forma, ajudar a superar esse difícil desafio.
\end{abstract}

Palavras-chave: Disfunção Cognitiva. Cuidado da Criança. Transtornos Reativos da Criança. Crianças com Deficiência. Transtornos do Neurodesenvolvimento.

\begin{abstract}
Children with cognitive disabilities are at increased risk of experiencing pain. In addition, it has been shown that this pediatric population often receives inadequate pain management. Pain is often very difficult to assess, especially in a defined subgroup with intellectual, non-verbal or even severe cognitive impairment. Although self-report is an alternative for a small number of children with less intellectual impairment, observational measures have the most consistent evidence to support their use of behavioral scales. Consequently, several observational pain assessment tools have been proposed to overcome this problem. Due to the absence of an ideal assessment tool, accurate pain assessment requires, after a case-by-case analysis, the selection of a more appropriate instrument or an association of instruments. The aim of this paper is to provide a review of the pain assessment instruments and scales commonly used in children with cognitive disabilities. It is hoped that with a critical discussion of each scale's own resources as well as its clinical applicability, they can somehow help to overcome this difficult challenge.
\end{abstract}

Keywords: Cognitive Dysfunction. Child Care. Child Reactive Disorders. Disabled Children. Neurodevelopmental Disorders.

\section{Introdução}

Crianças com deficiências cognitivas incluem condições clínicas como deficiências intelectuais, atrasos no desenvolvimento, incapacidade no desenvolvimento, dificuldades de aprendizagem e comprometimento cognitivo secundário a lesões cerebrais adquiridas ou doenças neurodegenerativas (BREAU et al., 2003). Outras condições clínicas, como por exemplo, crianças com distúrbios em diferentes espectros do autismo, também podem apresentar diferentes graus de deficiência cognitiva (CASCELLAM et al. 2019). O transtorno é, em geral, resultado de uma alteração no desempenho cerebral provocada por diferentes fatores: genéticos, distúrbios na gestação, problemas no parto ou na vida após o nascimento (LOBO, 2008). Crianças com deficiência intelectual, atualmente, sobrevivem em maior número e vivem por mais tempo (PATJA et al., 2000). O número de crianças nessa situação também está crescendo à medida que os avanços na tecnologia em Medicina aumentam as taxas de sobrevivência e a vida útil destas crianças (LORENZ et al., 1998). Segundo o IBGE, no censo de 2010 se contabilizou uma população de 2.611.536 portadores de deficiência intelectual. De acordo com a Associação Americana sobre Deficiência Intelectual do Desenvolvimento (AAIDD), as pessoas com deficiência intelectual têm dificuldades para aprender, entender e realizar atividades que são comuns e rotineiras para a maioria das pessoas. Dificuldades para aprender, entender e realizar atividades que são comuns e rotineiras para a maioria das pessoas com deficiência intelectual (LIANG; LI, 2019).

Crianças com deficiência intelectual podem apresentar uma maior sensibilidade dolorosa quando comparado às crianças "normais" em função de problemas físicos crônicos, a distúrbios complexos, por serem mais facilmente expostas a traumas e lesões e, também por passarem por um maior número de procedimentos médicos e/ou cirúrgicos (BREAU et al. 2003). De acordo com a Associação Internacional para o 
Estudo da Dor, a dor é uma experiência sensorial e emocional desagradável associada a danos teciduais reais ou potenciais, ou descrita em termos de tais danos. Muitos têm deficiências físicas e podem estar em alto risco de sofrer dores em decorrência de problemas médicos relacionados e tratamentos para esses problemas (NORDIN; GILLBERG, 1996). Seu perfil médico complexo também pode levar à dor sem tratamento por períodos mais longos, e ocorre porque os profissionais de saúde são desafiados a encontrar a causa de sua dor (BREAU; BURKITT, 2009). Aqueles com comprometimentos cognitivos graves podem também apresentar um maior risco de fatalidade em função de diagnósticos atrasados, porque a dor não é reconhecida (JANCAR; SPELLER, 1994). Por fim, essas crianças parecem ter maior risco de lesões acidentais (LELAND et al., 19940. Essa combinação de fatores levou a uma situação em que crianças com comprometimentos cognitivos graves têm maior probabilidade de sofrer dor do que outras crianças. Um estudo anterior (BREAU et al. 2003) avaliou, diariamente, a incidência de dor em crianças com comprometimento cognitivos graves, que revelou que $35 \%$ de uma amostra comunitária de crianças com deficiência intelectual que variava de moderada a profunda apresentava dor semanalmente, sendo que o tempo médio maior do que 9 horas por semana. Assim, acredita-se que crianças com deficiência intelectual sofram dores que poderiam ser mais bem gerenciadas pelos pais, cuidadores e profissionais da saúde em face do conhecimento atual (BREAU; BURKITT, 2009).

Em que pesem as dificuldades em se diagnosticar a manifestação da dor das crianças com deficiência intelectual, é extremamente importante incentivar uma melhor avaliação clínica associada aos instrumentos e métodos que permitem uma melhor detecção e monitoramento da dor (CARTER, 2020). Dessa forma, a avaliação da dor neste perfil de pacientes deve dar um passo além da subjetividade, visando sua completude ao incorporar no diagnóstico alguns instrumentos que explorem a realidade destas crianças, que apresentam uma complexidade física e emocional. Pais e os cuidadores são os quem melhor conhecem os portadores de deficiência intelectual e, de certa forma, sabem detectar as alterações da expressão facial, do comportamento, das vocalizações dos mesmos (CARTER, 2020; PARKER; BELEW, 2013). De forma geral, se pressupõe certa correspondência entre a experiência da dor e a capacidade de expressar a dor, o que tem sido problemático para aqueles que não conseguem se expressar verbalmente, como crianças pequenas e bebês (ANAND; CRAIG, 1996). Além da dor, estes pacientes podem ainda manifestar medo e desconforto durante os procedimentos médicos ou odontológicos. Trata-se de experiências sensitivas e emocionais, muitas vezes, desagradáveis aos pacientes, que podem estar associadas ou relacionadas a lesões reais ou potenciais dos tecidos. Estas experiências são particularmente traumatizantes para crianças com deficiência intelectual, que carecem do estabelecimento de vínculos, de referências, de relação de confiança e de identidade com os profissionais da saúde, para que transmitam segurança e melhoria na qualidade da atenção à saúde delas.

Em função do fato de que crianças com deficiência intelectual apresentarem habilidades verbais severamente limitadas, essas crianças têm sido excluídas da maioria dos estudos sobre dor pediátrica, já que o autorrelato da dor é considerado o padrão-ouro (BREAU et al., 2003). Muitas destas crianças apresentam deficiência visual ou auditiva, sendo que algumas delas podem apresentar comportamentos autistas. Estas condições físicas podem dificultar a interpretação de seus comportamentos e, de certa forma, interferem no desenvolvimento de ferramentas de observação para medir sua dor (MAURER; NEWBROUGH, 1987; PARKER; BELEW, 2013). Isso também significa que ferramentas de análise da dor, que são baseadas em comportamentos mostrados pela maioria das crianças, podem não ser adequadas para as crianças com deficiência intelectual (BREAU, 2003). Consequentemente, várias ferramentas observacionais de avaliação da dor foram propostas para superar esse problema. Em função da ausência de uma ferramenta de medição ideal, a avaliação precisa da dor requer, após uma análise caso a caso, a seleção da ferramenta mais apropriada ou de uma variedade de instrumentos combinados (CASCELLA et al., 2019).

Apesar de existirem instrumentos de avaliação da dor para crianças com deficiência intelectual, essas ferramentas nem sempre são utilizadas no dia a dia dos consultórios e clínicas (BREAU; BURKITT, 2009). Além disso, profissionais de saúde e prestadores de cuidados podem não estar familiarizados com os diferentes instrumentos de diagnóstico disponíveis (CASCELLA et al., 2019). Consequentemente, o uso inadequado ou limitado de ferramentas para avaliação da dor neste subgrupo pediátrico específico contribui para o manejo inadequado da dor (CARTER, 2020). Além disso, a dor mal administrada ou negligenciada pode levar a consequências deletérias na saúde física e na qualidade de vida destas crianças, por exemplo, que desencadeiam comportamentos auto ou heteroagressivos (CARR; OWENDESCHRYVER, 2007).

O presente estudo tem por objetivo revisar, de forma abrangente, os instrumentos de avaliação da dor comumente usados em crianças com deficiência cognitiva. O objetivo não é necessariamente esgotar as possibilidades de discussão sobre o assunto, mas contribuir para somar esforços com outros trabalhos já desenvolvidos sobre esse tema. 


\section{Desenvolvimento}

\subsection{Metodologia}

Trata-se de uma discussão sobre o uso de instrumentos de avaliação da dor para crianças com deficiências cognitivas. Nesse sentido, considerando-se o seu quadro teórico e objetivo, a metodologia de artigo discute criticamente as características e aplicabilidade destes instrumentos e como esses podem ajudar a superar esse difícil desafio, estimulando novas pesquisas destinadas a identificar a ferramenta de avaliação da dor mais válida e confiável. Dessa forma, o presente estudo é uma pesquisa descritiva, qualitativa, de revisão de literatura, que buscou na essência dos artigos pesquisados para melhor compreensão destes instrumentos e sua relevância no papel de pais, de cuidadores e de profissionais da saúde na avaliação da dor em pacientes com deficiência cognitiva. Os critérios de inclusão foram artigos, portarias e resoluções publicados no período de 1990 a 2020 e que fazem referência aos instrumentos de avaliação da dor neste grupo de crianças acometidas por deficiência cognitiva. Para fundamentar o estudo se buscaram artigos na literatura nas bases de dados da SciELO, Lilacs e Medline. Como critérios de inclusão seoptou por publicações até 2020, em português e em inglês, que estivessem relacionadas com o tema a partir dos descritores inseridos no DeCS Descritores em Ciências da Saúde (http://decs.bvs.br/), sendo esses: Criança, Disfunção Cognitiva, Cuidado da Criança, Transtornos Reativos da Criança, Crianças com Deficiência e Transtornos do Neurodesenvolvimento. Os critérios de exclusão foram: teses, livros, capítulos de livros, anais de congressos ou conferências, relatórios técnicos e científicos e, ainda documentos governamentais e/ou ministeriais. A busca resultou, inicialmente, da leitura do resumo dos artigos encontrados nas diferentes bases de dados, que responderam ao problema da pesquisa, totalizando 13 artigos. Destes 13 artigos selecionados, inicialmente, selecionaram-se 10 artigos após a leitura do texto completo, se enquadrando nos critérios de inclusão/exclusão.

\subsection{Discussão}

A mensuração da dor é um pré-requisito para um adequado tratamento analgésico em crianças com comprometimento cognitivo, mas é frequentemente prejudicado em função de suas habilidades limitadas de comunicação, múltiplos problemas complexos, que levam à manifestação da dor e, também em função de comportamentos "desadaptativos" em crianças acometidas por esta deficiência (MASSARO et al., 2014). A avaliação da dor em crianças com deficiência intelectual, não verbais ou em crianças com comprometimento cognitivo é indiretamente avaliada por observação das alterações fisiológicas (respiração, cor, tremores, sudorese, micção, pressão arterial e batimentos cardíacos) ou dos comportamentos potencialmente relacionados à dor (por exemplo, tipos de choro, expressões faciais ou verbais, comportamento agressivo e postura ou movimento corporal) (CASCELLA et al., 2019).

Diferentes escalas padronizadas foram desenvolvidas coletando vários conjuntos de potenciais indicadores de dor. As escalas mais usadas foram estruturadas com um número diferente de itens, com base apenas em respostas comportamentais ou a partir de uma combinação de alterações fisiológicas associadas às respostas comportamentais. De forma geral, são referidas como escalas observacionais de avaliação da dor. Uma estratégia muito eficaz, adotada por várias ferramentas de observação, demanda uma avaliação da dor na criança por parte dos pais. De forma geral, os pais estão mais familiarizados com o comportamento geral de seus filhos do que uma equipe médica, que geralmente não tem experiência anterior com a criança (CASCELLA et al., 2019). Em um estudo anterior (CARTER et al., 2017) se destacou que as mães se tornam especialistas em avaliar a dor de seus filhos sem a assistência de profissionais de saúde. Por outro lado, verificou-se em outro estudo que pais tendem a superestimar os sintomas em condições de dor aguda, enquanto eles podem subestimá-lo no cenário de doenças crônicas (VOEPELLEWIS et al., 2005).

Dessa forma, da análise das publicações se pode verificar que foram propostos inúmeros instrumentos para avaliação e monitoramento da dor em crianças com deficiência cognitiva, tais como: a escala Échelle Douleur Enfant San Salvador (DESS), o Perfil de Dor Pediátrica (Paediatric Pain Profile), o "check list" de Verificação de Dor em Crianças Não Comunicantes (NCCPC), o Indicador de Dor em Crianças com Deficiência Comunicativa e, ainda, a CHEOPS (de Children's Hospital of Eastern Ontario Pain Scale) (BREUA et al., 2000; DEFRIN et al., 2006; GARCIA; FERNANDES, 2007; GIUSIANO et al., 1995; HUNT et al., 2004; MASSARO et al., 2014; STALLARD et al., 2002). Outros instrumentos foram também propostos com o mesmo objetivo como a escala Rosto, Pernas, Atividade, Choro, Consolabilidade (Face, Legs, Activity, Cry and Consolability - FLACC), inicialmente proposta por Manworren e colaboradores (MANWOEEN; HYNAN, 2003) e, posteriormente, revisada e validada (r-FLACC) por outros autores (MALVIYA et al., 2006; MERKEL et al., 1997). A escala NCCPC também posteriormente revisada (NCCPC-R) (BREAU et al, 2002a, 2002b).

No Quadro 1 a seguir estão descritos os principais instrumentos de avaliação da dor em crianças com deficiência cognitiva, a forma de validação e respectivas publicações selecionadas no presente estudo, que se enquadraram nos critérios de inclusão. 
Quadro 1 - Instrumentos de avaliação da dor para crianças com deficiência cognitiva não verbal

\begin{tabular}{|c|c|c|c|}
\hline & Evidências / Comentários & Forma de Validação da Escala & \begin{tabular}{|l} 
Publicação que \\
Validou a Escala
\end{tabular} \\
\hline PPP & $\begin{array}{l}\text { Inclui a avaliação de comportamentos prejudiciais. Útil } \\
\text { para crianças que sofrem de dor crônica ou recorrente. } \\
\text { Sensibilidade de } 1,0 \text { e especificidade de } 0,91 \text {. }\end{array}$ & \begin{tabular}{|l|} 
Escala validada por meio de gravação em \\
vídeo das observações obtidas por pais e \\
pesquisadores. Observaram uma correlação \\
entre escores e concentração analgésica em \\
amostras de saliva coletadas de crianças.
\end{tabular} & Hunt et al. (2007) \\
\hline $\mathrm{PBC}$ & $\begin{array}{l}\text { Expressões faciais são identificadas como os } \\
\text { indicadores mais sensíveis da intensidade da dor. } \\
\text { Embora menos precisa, a escala reduzida deve ser } \\
\text { preferida por exigir menos tempo para concluir a } \\
\text { análise. Confiabilidade inter-examinador alta. }\end{array}$ & $\begin{array}{l}\text { Entrevistas com avaliadores antes do } \\
\text { tratamento ou reabilitação. Comparação com } \\
\text { medidas de autorrelato de dor. }\end{array}$ & $\begin{array}{l}\text { Kerns et al. } \\
\quad(1991)\end{array}$ \\
\hline NCCPC & $\begin{array}{l}\text { Prestadores de cuidados primários (pais, pais adotivos, } \\
\text { profissionais de saúde) podem utilizar esta escala. }\end{array}$ & $\begin{array}{l}\text { Indivíduos com comprometimento cognitivo } \\
\text { (idades de } 3 \text { a } 44 \text { anos) }(\mathrm{n}=54) \text {. }\end{array}$ & $\begin{array}{l}\text { Breau et al. } \\
(2000)\end{array}$ \\
\hline NCCPC-r & $\begin{array}{l}\text { Os cuidadores usaram a escala durante um período de } \\
\text { dor e outro sem dor. }\end{array}$ & $\begin{array}{l}\text { Treinamento não obrigatório. A lista } \\
\text { de verificação é relativamente extensa. } \\
\text { Atividade facial, choro e itens fisiológicos } \\
\text { se correlacionam com a pontuação geral, } \\
\text { enquanto movimentos sociais / emocionais, } \\
\text { parte do corpo ferida e atividades da vida } \\
\text { diária não. }\end{array}$ & $\begin{array}{l}\text { Breau et al. } \\
(2002 a)\end{array}$ \\
\hline $\begin{array}{l}\text { NCCPC- } \\
\text { PV }\end{array}$ & $\begin{array}{l}\text { O pequeno tamanho da amostra e sua restrição às } \\
\text { crianças gravemente prejudicadas limitam seu uso } \\
\text { a subgrupos específicos (Versão pós-operatória). } \\
\text { Alta confiabilidade inter observadores. Fornece } \\
\text { mais informações sobre a gravidade da dor do que o } \\
\text { NCCPC-r. }\end{array}$ & $\begin{array}{l}\text { Um cuidador e um pesquisador observaram } \\
\text { crianças por } 10 \text { minutos antes e após a } \\
\text { cirurgia. Correlação com a classificação de } \\
\text { EVA baseada na intensidade da dor da criança. }\end{array}$ & $\begin{array}{l}\text { Breau et al. } \\
(2002 a)\end{array}$ \\
\hline DESS & $\begin{array}{l}\text { A escala DESS constitui um instrumento com grande } \\
\text { potencial para a avaliação objetiva da dor em criança } \\
\text { com deficiência profunda. O reconhecimento dos sinais } \\
\text { de dor nestas crianças exige o conhecimento prévio das } \\
\text { características e comportamentos habituais da criança, } \\
\text { que só os prestadores de cuidados habituais - pais, } \\
\text { educadores, terapeutas - possuem. A escala DESS se } \\
\text { mostra promissora, mas mais estudos são necessários } \\
\text { em crianças com deficiência intelectual para que possa } \\
\text { ser recomendado. }\end{array}$ & $\begin{array}{l}\text { A validação da escala DESS foi realizada } \\
\text { apenas em francês. Em 2007, Garcia e } \\
\text { Fernandes (2007) publicaram um artigo } \\
\text { em português, sendo que estudos são ainda } \\
\text { necessários para validar a escala em inglês. } \\
\text { Estas autoras salientaram que a escala DESS } \\
\text { favorece a análise da intensidade da dor em } \\
\text { crianças com precisão. }\end{array}$ & $\begin{array}{l}\text { Giusiano et al. } \\
\text { (1995) }\end{array}$ \\
\hline FLACC & $\begin{array}{l}\text { Escala observacional simples, visando avaliar a } \\
\text { dor em crianças incapazes de verbalizar a presença } \\
\text { ou gravidade da dor. Inclui cinco categorias de } \\
\text { comportamentos de dor: expressão facial, movimento } \\
\text { das pernas, atividade, choro e consolabilidade. }\end{array}$ & $\begin{array}{l}\text { Escala validada por meio da análise de grupo } \\
\text { de crianças que receberam opioides em relação } \\
\text { ao controle (não tratado). }\end{array}$ & $\begin{array}{l}\text { Merkel et al } \\
\text { (1997) }\end{array}$ \\
\hline r-FLACC & $\begin{array}{l}\text { Escala revisada apropriada para avaliação da dor } \\
\text { aguda em crianças com necessidades especiais e para } \\
\text { crianças afetadas por deficiências neurológicas graves, } \\
\text { como paralisia cerebral. }\end{array}$ & $\begin{array}{l}\text { Avaliação de dor autorreferida. Dois } \\
\text { avaliadores pontuaram a dor pós-operatória } \\
\text { antes e após uso de analgésico. As observações } \\
\text { foram filmadas e avaliadas por um revisor } \\
\text { cego. }\end{array}$ & $\begin{array}{l}\text { Malviya et al. } \\
\quad(2006)\end{array}$ \\
\hline INRS & $\begin{array}{l}\text { Pouco utilizada e avaliada em estudos. Trata-se de } \\
\text { uma adaptação da escala de classificação numérica que } \\
\text { incorpora as descrições dos pais e/ou cuidadores das } \\
\text { respostas passadas e atuais de seus filhos à dor. }\end{array}$ & $\begin{array}{l}\text { Pais, enfermeiros e assistentes de pesquisa } \\
\text { estavam envolvidos. NCCPC-PV usado para } \\
\text { comparação. Observou-se uma correlação } \\
\text { de moderada a forte }(0,63-0,73) \text { entre as } \\
\text { classificações do INRS e as de NCCPC-PV. }\end{array}$ & $\begin{array}{l}\text { Solodiuk et al. } \\
\quad(2010)\end{array}$ \\
\hline PICIC & $\begin{array}{l}\text { Pouco utilizado. Apresenta limitações. Observada } \\
\text { relação significativa entre três dos seis tópicos } \\
\text { avaliados e a ocorrência de severidade da dor. }\end{array}$ & $\begin{array}{l}\text { Um dos parâmetros foi o preditor mais forte } \\
\text { e, por si só, classificou corretamente } 87 \% \text { dos } \\
\text { episódios de dor e de ausência de dor. }\end{array}$ & $\begin{array}{l}\text { Stallsrg et al. } \\
\qquad(2002)\end{array}$ \\
\hline CHEOPS & $\begin{array}{l}\text { Trata-se de uma escala comportamental para avaliar } \\
\text { a dor pós-operatória em crianças pequenas. Pode ser } \\
\text { usado para monitorar a eficácia das intervenções para } \\
\text { reduzir a dor e o desconforto. A escala inclui seis } \\
\text { categorias de comportamento da dor: (choro, facial, } \\
\text { verbal, torso, tato e pernas). Uma pontuação variando } \\
\text { de } 0 \text { a } 2 \text { ou } 1 \text { a } 3 \text { é atribuída a cada atividade e a } \\
\text { pontuação total varia entre } 4 \text { e } 13 \text {. }\end{array}$ & $\begin{array}{l}167 \text { crianças com idades de } 1 \text { a } 5,5 \text { anos } \\
\text { foram filmadas antes e após a cirurgia, } \\
\text { antes da analgesia na unidade de cuidados } \\
\text { pós-anestésicos e na enfermaria. Quatro } \\
\text { observadores classificaram o comportamento } \\
\text { da dor. O estudo validou a escala CHEOPS cuja } \\
\text { análise mostrou uma excelente confiabilidade } \\
\text { inter-examinadores e intra examinadores ( }> \\
0,9 \text { e }>0,8 \text {, respectivamente). }\end{array}$ & $\begin{array}{l}\text { Suraseranivongse } \\
\text { et al. }(20010\end{array}$ \\
\hline
\end{tabular}

FLACC: Faces, Legs, Activity, Cry and Consolability; INRS: Individualized Numerical Rating Scale; NCCPC: Non-communicating Children's Pain Checklist; NCCPC-PV: Non-communicating Children's Pain Checklist-Postoperative Version; NCCPC-r, NCCPC revisado; NRS: Numeric Rating Scale; PBC: Pain Behaviour Checklist; PPP: Paediatric Pain Profile; r-FLACC: FLACC revisado; EVA: escala visual analógica; PICIC: pain indicator for communicatively impaired children; CHEOPS: Children's Hospital of Eastern Ontario Pain Scale.

Fonte: dados da pesquisa. 
Alguns estudos compararam diferentes instrumentos, embora certas escalas apresentem especificidades. Em um estudo prévio (MASSARO et al., 2014), foram comparadas três escalas observacionais de dor: a escala DESS, a única ferramenta que foi validada especificamente para pessoas com paralisia cerebral, o NCCPC, a escala mais citada na literatura para crianças com problemas cognitivos não verbais e a escala CHEOPS, que não é específica. As escalas DESS e NCCPCPV são validadas para pacientes com comprometimento cognitivo para uma ampla faixa etária. Foram comparadas as concordâncias entre avaliadores, a complexidade de uso e as opiniões dos cuidadores sobre a adequação de cada escala para medir a dor. Da análise dos resultados se observou que a escala NCCPC é adequada e fácil de ser utilizada para avaliação da dor em crianças com comprometimento cognitivo em um ambiente clínico. A escala DESS apresentou a melhor concordância de resultados entre os avaliadores, mas foi a escala que mais frequentemente exigia informações do cuidador. Isso se deve ao fato de a escala DESS exigir que os itens sejam classificados em relação ao comportamento típico da criança, tornando a ferramenta menos simples de usar, quando os observadores não estão familiarizados com a criança. Por outro lado, a escala CHEOPS foi o instrumento em que os avaliadores precisaram de um menor número de informações a respeito da criança avaliada para o preenchimento e realizar a pontuação.

Em uma revisão da literatura sobre o assunto se afirmou que inúmeros esforços têm sido realizados para gerar instrumentos validados, que sejam confiáveis e para avaliação da dor em crianças com deficiência intelectual desde os anos 1990. Os autores enfatizaram ainda que há poucas publicações em relação à efetividade de instrumentos projetados para crianças com deficiências cognitivas. Diferentes instrumentos de observação foram desenvolvidos para avaliar a dor em crianças. Dependendo da idade da criança e de sua capacidade de se comunicar com o prestador de cuidados, existem muitos instrumentos confiáveis e validados, adequados para diferentes contextos clínicos (BREAU; BURKITT, 2009). Cada ferramenta possui recursos adequados para condições específicas. Por exemplo, algumas dessas escalas são mais válidas para avaliar a dor aguda (por exemplo, dor pós-operatória ou dor decorrente de cuidados médicos). Outras escalas, por outro lado, são mais adequadas para a investigação de condições de dor crônica, que geralmente são acompanhadas por algumas respostas adaptativas, que incluem ansiedade e transtornos de humor (CARTER, 2020).

De acordo com um artigo de revisão (CASCELLA et al., 2019), afirmou-se que crianças com deficiência cognitiva correm maior risco de ter experiência de dor; no entanto, neste grupo de crianças vulneráveis, muitas vezes, o manejo da dor é inadequado. Ainda segundo estes autores, os instrumentos de avaliação da dor usadas em crianças sem deficiência cognitiva podem não ser precisos em crianças com deficiência no neurodesenvolvimento; cada escala proposta mostra recursos adequados para condições clínicas específicas; as escalas são mais adequadas para avaliar a dor aguda (por exemplo, dor pós-operatória ou dor decorrente de cuidados médicos), enquanto outras ferramentas para avaliar as condições de dor crônica estão também disponíveis (BREAU; BURKITT, 2009). As famílias, geralmente, avaliam corretamente a dor da criança; esforços devem ser feitos para treinar os profissionais de saúde (por exemplo, pediatras, neuropsiquiatras infantis, enfermeiros, psicólogos e fisiatras) e melhorar a avaliação da dor em crianças com necessidades especiais. Além disso, o envolvimento dos prestadores de cuidados no uso de escalas de dor deve ser mais enfatizado (CARTER, 2020). Dessa forma, uma cuidadosa avaliação da dor geralmente é possível apenas com o uso de ferramenta apropriada após uma análise caso a caso ou montando uma combinação de vários métodos Apesar deste apelo, há um consenso de que não existe uma escala ideal para avaliar a dor em crianças com deficiência cognitiva. No Quadro 2 estão descritas as características ideais que um instrumento deveria apresentar.

Quadro 2 - Características da ferramenta ideal para avaliar a dor em crianças com deficiências cognitivas

1- Simples e facilmente assimilável à prática diária por profissionais de saúde (ou seja, enfermeiros, médicos, dentistas e fisioterapeutas), bem como pelos pais e cuidadores.

2- Aplicável em ambientes domésticos e institucionais.

3- Adequado para dores de etiologia variada, como agudas (por exemplo, relacionadas à lesões, procedimentos cirúrgicos ou médicos) e condições de dor crônica.

4- Completo, cobrindo comportamentos específicos relacionados à dor (por exemplo, comportamentos auto lesivos ou respostas defensivas de congelamento -"freezing reaction") e alterações fisiológicas, embora não duradouro.

5- Útil para cada nível de comprometimento cognitivo e limitação física.

6- Incluir a contribuição dos pais, quando possível. Fonte: Adaptado de Cascella et al. (2019).

A equipe de saúde, como enfermeiros e clínicos, bem como gestores, educadores, líderes e pesquisadores devem contribuir efetivamente para garantir que a avaliação e o gerenciamento da dor sejam realizados da melhor forma (CARTER, 2020). Embora evidências para validar as diferentes escalas sejam necessárias, a avaliação da dor em crianças com comprometimento cognitivo profundo com as escalas disponíveis é extremamente apropriada e, de forma geral, oferecem uma clara direção sobre a avaliação da dor. Apesar de nenhuma das escalas disponíveis serem completas, idealmente é importante avaliar a ocorrência da dor em crianças, especialmente não verbais, utilizandose ferramentas específicas que foram validadas. Tudo isso precisa ser feito em diálogo com os pais/responsáveis e cuidadores da criança. Embora os dados suficientes para julgar a validade e a confiabilidade de algumas das escalas 
disponíveis sejam limitados no momento, os interessados devem ser incentivados a buscarem informações sobre as evidências em andamento para apoiar o seu uso à medida que novos estudos são publicados.

De forma geral, é recomendado que a avaliação da dor em crianças com deficiência intelectual deve seguir os mesmos princípios de outros grupos (BREAU; BURKITT, 2009). Embora o autorrelato seja a melhor e a mais direta forma de se obter informações sobre a intensidade da dor se a criança for capaz de fazê-lo enquanto a sofre, não há evidências para apoiar o autorrelato como padrão para muitas dessas crianças. Quando possível, uma avaliação completa da dor deve incluir alguma estimativa do nível intelectual da criança. Nesse sentido, uma avaliação multiprofissional é de extrema importância. Os resultados dos testes formais podem ser utilizados para se determinar se é provável que a criança seja capaz de, considerando-se a possibilidade de um autorrelato, o que seria importante para complementar uma análise da dor. Na prática, com base em situações hipotéticas, esta poderia ser a melhor maneira de avaliar a capacidade da criança de usar o autorrelato. Porém muitas vezes, na rotina diária, as crianças com deficiência cognitiva, provavelmente, não serão capazes de relatar uma dor real. A escala NCCPC-PV é indicada para ser utilizada quando o autorrelato não for possível ou quando houver preocupações sobre a capacidade de autorrelato de uma criança. Quando a dor crônica ou recorrente é possível também associar a utilização de diferentes escalas para avaliar a dor, especialmente, em casos em que são necessárias informações sobre padrões temporais de dor e podem ajudar a desvendar mudanças eventuais comportamentais decorrentes de dores relacionadas com outros fatores, como algumas doenças ou problemas de sono. Desenvolvendo um registro do histórico de dor da criança, usando um formato como o fornecido com o Pediatric Pain Profile (BREAU; BURKITT, 2009), também pode ser útil em casos de dor crônica ou múltipla ou para detectar novos problemas emergentes de dor. Algumas escalas podem também serem utilizadas para avaliar mudanças de comportamento, como autoagressão ou outras manifestações para verificar se a dor está presente, em que local pode estar localizada e em que medida a sintomatologia dolorosa afeta o comportamento da criança. Nesse sentido, a escala DESS, desenvolvida por uma equipe do Hospital "San Salvadour", em Hyères, França, constitui um instrumento que possibilita a quantificação e monitorização da dor em crianças com limitações verbais, cognitivas e comportamentais (GARCIA; FERNANDES, 2007). Apesar desta escala exigir um conhecimento prévio das características e comportamentos habituais da criança com deficiência cognitiva para o reconhecimento dos sinais de dor, geralmente, realizado pelos cuidadores habituais (principalmente pais, educadores e terapeutas), esta escala permite adotar uma linguagem comum entre cuidadores e profissionais de saúde na identificação dos sinais de dor, o que de certa forma colaboram para um melhor controle da dor nestas crianças (GARCIA; FERNANDES,
2007).

A aplicação da escala de dor é um instrumento facilitador para os profissionais e também para os pais e cuidadores. Por meio da escala, a dor do assistido poderá ser avaliada e ponderada de maneira real e aplicada. Por outro lado, a percepção da manifestação da dor por profissionais da saúde em crianças com limitações cognitivas e neuromusculares com vistas identificação das necessidades de intervenção nestes pacientes pode ser prejudicada por causa da falta de conhecimentos sobre o assunto. Nesse sentido, é imperativo que estas diferentes escalas sejam divulgadas por meio de treinamentos em que se observem as suas vantagens, limitações e funções. É sabido que cada pessoa lida com a dor de alguma forma, e que este sentimento é projetado em função da forma como os pacientes podem lidar com esta mesma dor, especialmente, para aqueles que não conseguem expressá-la. A escala é, certamente, um parâmetro mais seguro e eficaz de transmitir uma informação mais fidedigna do real sentido e avaliação da dor. A escala deve ser corretamente indicada, ter um fácil acesso e ser autoexplicativa quanto sua aplicação, para que aqueles que cuidam de crianças com deficiência cognitiva possam utilizá-la para melhor compreendê-la e utilizála. Dessa forma, fica favorecido o estabelecimento de um maior vínculo, estabelecendo-se a referência, uma relação de confiança, de identidade e de melhoria na qualidade da atenção a saúde para estes pacientes especiais. Dessa forma, propõese a formação continuada de profissionais da saúde sobre a dor e a aplicação da escala DESS em crianças com limitações cognitivas e neuromusculares. Uma forma de divulgação dos diferentes instrumentos de avaliação da dor em pacientes com deficiência cognitiva poderia ser consideradas por meio de estratégias, a partir de um formato eletrônico em um sítio na rede mundial de computadores, em um meio verdadeiramente interativo por instituições, que atendem pacientes com esta condição clínica, atendendo as necessidades educacionais específicas ou através de treinamento em ambientes virtuais de saúde (POLASSI et al., 2018) para pais, cuidadores e trabalhadores da saúde em geral.

Finalmente, novas pesquisas sugerem que crianças com deficiência intelectual usam diferentes estratégias de enfrentamento quando sentem dor. Assim, a avaliação por parte de pais e/ou cuidadores de como a criança lida com a dor pode ajudar a orientar a criança a usar sua estratégia mais eficaz, ou tentar novas estratégias se as estratégias comumente utilizadas não forem mais efetivas para elas. Embora haja alguma indicação de que crianças com deficiência intelectual pode fornecer dados para compor um histórico sobre seu enfrentamento da dor quando a dor não estiver se manifestando, o relato dos pais também pode ser a maneira mais válida de entender as estratégias e as habilidades de enfrentamento de uma criança nos diferentes momentos de manifestação da dor.

\section{Conclusão}

O presente estudo mostrou que a dor é uma questão 
significativa em crianças com deficiência cognitiva em decorrência de vários problemas de saúde e aos inúmeros procedimentos dolorosos a que são submetidos por profissionais médicos e não médicos. A avaliação e o manejo da dor são, frequentemente, complicados por barreiras de comunicação. Assim, mesmo que o manejo adequado desse problema tenha um objetivo terapêutico primário, muitas vezes é inadequado. De fato, a dor é um dos sintomas que não é rotineiramente investigado em função do fato de que a avaliação em crianças com deficiência cognitiva seja, muitas vezes, bastante difícil. Além disso, ferramentas comumente utilizadas para avaliar a dor em crianças "normais" podem não ser confiáveis em condições específicas como a deficiência cognitiva e os profissionais da saúde, geralmente, não estão familiarizados com os diferentes instrumentos de diagnóstico disponíveis. Para superar esses problemas, várias ferramentas específicas foram produzidas. Crianças com comprometimento cognitivo leve, de modo geral, devem ter a chance de usar uma escala de autorrelato se os profissionais de saúde tiverem certeza de que elas compreendem completamente e de que elas são capazes de utilizar a escala proposta. Por outro lado, em crianças não comunicativas com comprometimento cognitivo, as ferramentas observacionais de avaliação da dor representam o padrão-ouro para identificar a dor. No entanto, como não há ferramenta de medição ideal para essa população, uma avaliação precisa da dor, geralmente, é possível apenas com o uso da ferramenta mais apropriada, após uma análise caso a caso ou com uma variedade de métodos combinados. Com base nestas premissas, mais estudos são necessários para desenvolver e validar novos instrumentos e para melhorar as ferramentas já em uso, uma vez que a avaliação da dor nesta população requer habilidades e conhecimentos específicos.

\section{Referências}

ANAND, K.J.; CRAIG, K.D. New perspectives on the definition of pain. Pain, v.67, n.1, p.3-6, 1996.

BREAU, L.M. Non-communicating children's pain checklist: better pain assessment for severely disabled children. Expert Rev Pharmacoecon Outcomes Res, v.3, n.3, p.327-339, 2003.

BREAU, L.M.; BURKITT, C. Assessing pain in children with intellectual disabilities. Pain Res Manag, v.14, n.2, p.116-20, 2009.

BREAU, L.M. et al. The incidence of pain in children with severe cognitive impairments. Arch. Pediatr. Adolesc. Med., v.157, n.12, p.1219-26, 2003.

BREAU, L.M. et al. Validation of the non-communicating children's pain checklist-postoperative version. Anesthesiology, v. 96, n. 3, p. $528-35,2002$ a.

BREAU, L.M. et al. Preliminary validation of an observational pain checklist for persons with cognitive impairments and inability to communicate verbally. Dev. Med. Child. Neurol., v. 2, n.9, p.609-616, 2000.

BREAU, L.M. et al. Psychometric properties of the noncommunicating children's pain checklist-revised. Pain, v.99, n.1/2, p.349-357, 2002b.
CARR, E.G.; OWEN-DESCHRYVER, J.S. Physical illness, pain, and problem behavior in minimally verbal people with developmental disabilities. J. Autism Dev. Disord., v.37, n.3, p.413-24, 2007.

CARTER, B. Communicating pain: the challenge of pain assessment in children with profound cognitive impairment. Compr Child Adolesc, Nurs., v. 43, n. 1, p. 10-14, 2020.

CARTER, B. et al. Developing a sense of knowing and acquiring the skills to manage pain in children with profound cognitive impairments: mothers' perspectives. Pain Res Manag., v.2017, p.2514920, 2017.

CASCELLA, M. et al. The challenge of pain assessment in children with cognitive disabilities: Features and clinical applicability of different observational tools. J. Paediatr. Child Health, v.55, n.2, p.129-135, 2019.

DEFRIN, R.; LOTAN, M.; PICK, C.G. The evaluation of acute pain in individuals with cognitive impairment: a differential effect of the level of impairment. Pain, v.124, n.3, p.312-320, 2006.

GARCIA, M.; FERNANDES, A. Avaliação da dor nas crianças com deficiência profunda: a escala DESS. Referência, v. 2, n. 5, p. 17-22, 2007.

GIUSIANO, B. et al. Utilization of neural network in the elaboration of an evaluation scale for pain in cerebral palsy. Methods Inf. Med., v.34, n.5, p.498-502, 1995.

HUNT, A. et al. Clinical validation of the paediatric pain profile. Dev Med Child Neurol., v.46, n.1, p.9-18, 2004.

HUNT, A. et al. Development of the paediatric pain profile: role of video analysis and saliva cortisol in validating a tool to assess pain in children with severe neurological disability. J. Pain Symptom Manage, v.33, n.3, p.276-289, 2007.

JANCAR, J.; SPELLER, C.J. Fatal intestinal obstruction in the mentally handicapped. J. Intellect Disabil Res., v.38, p.413-422, 1994.

KERNS, R.D. et al. The Pain Behavior Check List (PBCL): factor structure and psychometric properties. J Behav Med., v. 14, n. 2, p.155-167, 1991 .

LELAND, N.L.; GARRARD, J.; SMITH, D.K. Comparison of injuries to children with and without disabilities in a day-care center. J Dev Behav Pediatr., v.15, n.6, p.402-408, 1994.

LIANG, F.; LI, P. Characteristics of cognitive in children with learning difficulties. Translational Neuroscie., v.10, p.141-146, 2019.

LOBO, I. Birth defects: prevention and treatment. Nature Education. 2008. Disponível em: <https://www.nature.com/ scitable/topicpage/birth-defects-causes-and-statistics-863/>. Acesso em: 16 mar 2020.

LORENZ, J.M. et al. A quantitative review of mortality and developmental disability in extremely premature newborns. Arch. Pediatr. Adolesc. Med., v.152, n.5, p.425-35, 1998.

MALVIYA, S. et al. The revised FLACC observational pain tool: improved reliability and validity for pain assessment in children with cognitive impairment. Paediatr. Anaesth., v.16, n.3, p.25865, 2006.

MANWORREN, R.C.; HYNAN, L.S. Clinical validation of FLACC: preverbal patient pain scale. Pediatr. Nurs., v.29, n.2, p.140-6, 2003.

MASSARO, M. et al. A comparison of three scales for measuring pain in children with cognitive impairment. Acta Paediatr., v.103, n.11, p.e495-500, 2014. 
MAURER, H.; NEWBROUGH, J. R. Facial expressions of mentally retarded and nonretarded children: II. Recognition by nonretarded adults with varying experience with mental retardation. Am. J. Ment. Defic., v. 91, n. 5, p. 511-5, 1987.

MERKEL, S. et al. The FLACC: a behavioral scale for scoring postoperative pain in young children. Pediatr Nurs., v.23, n.3, p.293-297, 1997.

NORDIN, V.; GILLBERG, C. Autism spectrum disorders in children with physical or mental disability or both. I: Clinical and epidemiological aspects. Dev. Med. Child Neurol., v.38, n.4, p.297-313, 1996.

PARKER, J.; BELEW, J.L. Qualitative evaluation of a pain intensity screen for children with severe neurodevelopmental disabilities. Pain Manag Nurs., v.14, n.4, p.e115-e123, 2013.

PATJA, K. et al. Life expectancy of people with intellectual disability: a 35-year follow-up study. J. Intellect Disabil Res. v.44, p.591-599, 2000.
POLASSI, M.R. et al. Uso de plataformas integradoras de ferramentas tecnológicas e pedagógicas em ambiente virtual de aprendizagem em profissões de saúde. Rev. Ens. Educ. Cienc Human., v. 9, n. 2, p. 168-176, 2018.

SOLODIUK, J.C. et al. Validation of the Individualized Numeric Rating Scale (INRS): a pain assessment tool for nonverbal children with intellectual disability. Pain, v.150, n.2, p.231-236, 2010.

STALLARD, P. et al. The development and evaluation of the pain indicator for communicatively impaired children (PICIC). Pain, v. 98, n. 1-2, p. 145-9, 2002.

SURASERANIVONGSE, S. et al. Cross-validation of a composite pain scale for preschool children within 24 hours of surgery. Bras. J. Anaesth., v.87, n.3, p.400-405, 2001.

VOEPEL-LEWIS, T.; MALVIYA, S.; TAIT, A.R. Validity of parent ratings as proxy measures of pain in children with cognitive impairment. Pain Manag Nurs., v.6, n.4, p.168-174, 2005. 